\title{
Charge asymmetry of top quarks
}

\author{
Paola Ferrario* \\ IFIC (UVEG - Consejo Superior de Investigaciones Científicas) \\ E-mail: paola.ferrariodific.uv.es
}

\section{Germán Rodrigo}

IFIC (UVEG - Consejo Superior de Investigaciones Científicas)

E-mail: german.rodrigodific.uv.es

The LHC is a promising machine to discover new physics in the top sector. There are several models that predict the existence of heavy colored resonances decaying to top quarks in the $\mathrm{TeV}$ energy range. The production of such resonances might generate a sizable charge asymmetry of top versus antitop quarks. At the Tevatron, a $2 \sigma$ discrepancy with the SM prediction for the forward-backward asymmetry has been found, boosting a renewed interest for this kind of models. We consider a toy model with general flavour dependent couplings of the resonance to quarks, of both vector and axial-vector kind and investigate the possible constraints on the coupling space from the measurement of the asymmetry and the invariant mass distribution at the Tevatron. Then, we define a central asymmetry in a specific kinematic region and investigate its signature at the LHC as well as its statistical significance, for exclusive processes.

XVIII International Workshop on Deep-Inelastic Scattering and Related Subjects April 19 -23, 2010

Convitto della Calza, Firenze, Italy

${ }^{*}$ Speaker. 


\section{Introduction}

The achievement of $7 \mathrm{TeV}$ in the centre-of-mass energy at the LHC has started off an exciting era for particle physics. An integrated luminosity of $1 \mathrm{fb}^{-1}$ is planned for the first 18 to 24 months of running, where precise measurements of the Standard Model (SM) will be performed as well as hints of new physics possibly unveiled. The LHC is a top machine: of the order of $10^{5}$ top antitop quark pairs will be produced already in the first running, with $1 \mathrm{fb}^{-1}$, which is a larger sample than at the Tevatron. With such a huge top statistic, it is natural investigating the reach of top production in order to find signals of new physics at the LHC. Several models predict the existence of heavy colored resonances decaying to top-antitop quark pairs that might be observed at the LHC, like axigluons in chiral color models, different kinds of massive gauge bosons in coloron and top color models or Kaluza Klein excitations in extra dimensional models. These resonances should be detectable in top-antitop quark events, particularly in those models where the coupling of the new gauge bosons to the third generation is enhanced with respect to the lighter fermions. Their existence modifies the $q \bar{q} \rightarrow t \bar{t}$ production cross-section, while gluon-gluon fusion to top quarks stays, at first order, unaltered, because a pair of gluons do not couple to a single extra resonance in this kind of models.

The natural signature of these resonances is a peak in the invariant mass distribution of the top-antitop quark pair located at the mass of the new resonance. However, asymmetries can be an alternative way of revealing these resonances. Some of those exotic gauge bosons, such as the axigluons, might generate already at tree-level a charge asymmetry through the interference with the $q \bar{q} \rightarrow t \bar{t}$ SM amplitude $[1,2]$.

QCD at tree level predicts that top-antitop quark pair production at hadron colliders is charge symmetric, namely the differential charge asymmetry, defined as:

$$
A(\cos \theta)=\frac{N_{t}(\cos \theta)-N_{\bar{t}}(\cos \theta)}{N_{t}(\cos \theta)+N_{\bar{t}}(\cos \theta)}
$$

vanishes for every value of $\theta$, where $N_{t(\bar{t})}(\cos \theta)$ is the number of top (antitop) quarks produced at a certain angle $\theta$ with respect to the incoming quark. Nevertheless, an asymmetry is generated at $\mathscr{O}\left(\alpha_{s}^{3}\right)$ from the graphs shown in Fig. 1 [3].
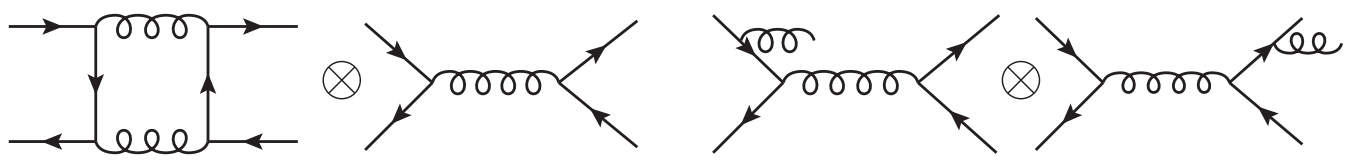

Figure 1: Graphs contributing to the QCD charge asymmetry in quark-antiquark production.

The charge asymmetry coming from the real hard radiation has opposite sign compared to the soft and virtual corrections and the latter is always larger than the former. On the other hand, $q g$ originated processes generate a contribution to the asymmetry much smaller than $q \bar{q}$, and $g g$ fusion is obviously symmetric. 


\section{The charge asymmetry at the Tevatron}

At the Tevatron, the charge asymmetry is equivalent to the forward-backward asymmetry, from CP invariance. The forward-backward asymmetry of top quarks has already been measured at the Tevatron proton-antiproton collider [4-6], at a center-of-mass energy of $\sqrt{s}=1.96 \mathrm{TeV}$. The most recent value for the forward-backward asymmetry in the laboratory frame, extracted with a luminosity of $3.2 \mathrm{fb}^{-1}$, is [6]:

$$
A_{\mathrm{FB}}^{p \bar{p}}=\frac{N_{t}(\cos \theta>0)-N_{t}(\cos \theta<0)}{N_{t}(\cos \theta>0)+N_{t}(\cos \theta<0)}=0.193 \pm 0.065_{\text {stat. }} \pm 0.024_{\text {syst. }},
$$

where $\theta$ is the angle between the top quark and the proton beam.

The total charge asymmetry generated at the Tevatron by QCD at NLO has been calculated to be $[1,7]$ :

$$
A=\frac{N_{t}(y \geq 0)-N_{\bar{t}}(y \geq 0)}{N_{t}(y \geq 0)+N_{\bar{t}}(y \geq 0)}=0.051(6),
$$

where $y$ is the quark (antiquark) rapidity in the laboratory frame. Comparing the theoretical prediction (2.2) with the experimental result (2.1), a discrepancy emerges of about two sigmas, that opens a window to the presence of new physics.

In a general, model independent way, we consider heavy color-octet boson resonances decaying to $t \bar{t}$ with arbitrary vector and axial-vector couplings to quarks $g_{V}^{q}$ and $g_{A}^{q}$. In the Appendix of Ref. [8] we list the differential cross section for $t \bar{t}$ production in the presence of such a resonance. A positive asymmetry can be generated if the term from the squared amplitude of the massive coloroctet dominates over the term of the interference. This is possible if the vector couplings are large enough. However, although the total cross section might still be compatible with the SM prediction in that case, because the contribution of the excited gluon is suppressed by powers of its mass, the top-antitop quark invariant mass distribution might be enhanced considerably, particularly for high values of the top-antitop quark invariant mass. In [9], the differential distribution in the invariant top-antitop quark mass is presented. The measurement is performed with $2.7 \mathrm{fb}^{-1}$ of integrated luminosity. The invariant mass is divided in nine bins and the last one $(0.8-1.4 \mathrm{TeV})$ is the most sensible to extra contributions beyond the $\mathrm{SM}$ at the $\mathrm{TeV}$ scale. The charge asymmetry and the invariant mass distribution probe different combinations of the vector and axial-vector couplings; therefore, by combining both limits, one can constrain complementary regions in the parameter space.

Our results are shown in Fig. 2 [10], where, for a given value of the mass of the color-octet resonance, we provide the allowed region at 95\% C.L. (for the flavor-universal scenario) and 90\% (for the other one) in the $g_{V}-g_{A}$ plane. The solid lines are obtained from the charge asymmetry, while the dashed lines are derived from the last bin of the invariant mass distribution. In the flavoruniversal case, the allowed regions are quite constrained; indeed, at 90\% C.L. we do not find any overlapping region for any value of the color-octet mass, and future experimental measurements with higher statistics can shrink significantly, or even exclude completely the allowed regions at $95 \%$ C.L.. If the axial coupling has different sign for light and top quarks, $g_{A}^{q}=-g_{A}^{t}$, we find that, for $\left|g_{A}\right|<2$ and independently of the resonance mass, the region about

$$
\left(\left|g_{A}\right|-2.3\right)^{2}+\left|g_{V}\right|^{2} \gtrsim 1.8^{2}
$$



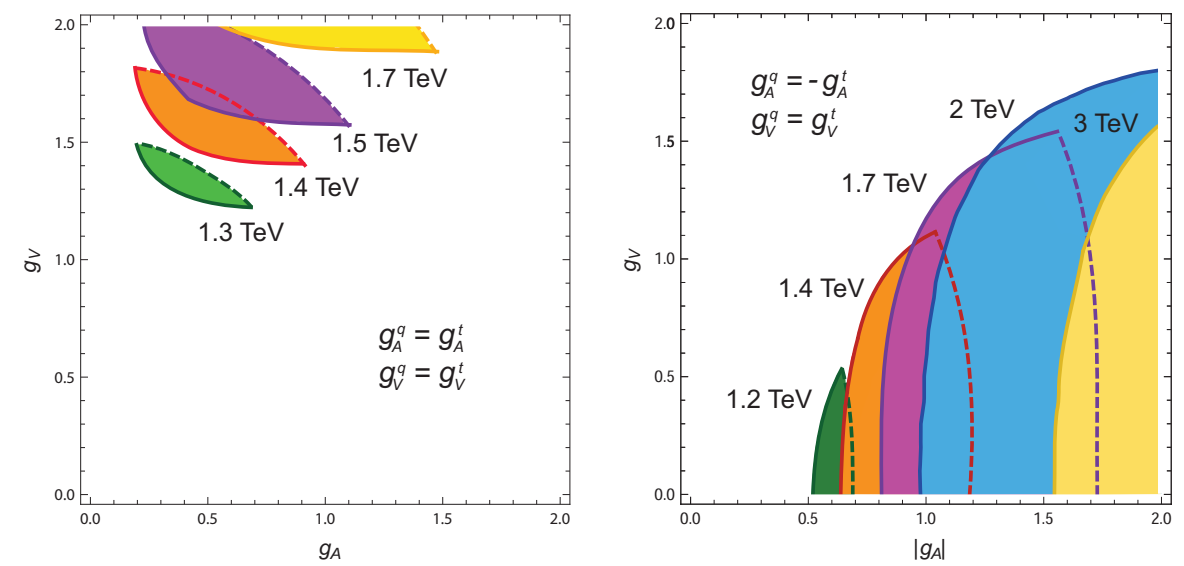

Figure 2: Constraints on the vector and axial-vector couplings for different values of the resonance mass. In the flavour-universal case (left plot) the contours are shown at $95 \%$ C.L., while in the other case (right plot) at $90 \%$ C.L.

is excluded at $90 \%$ C.L. Furthermore, for fixed values of the vector and axial-vector couplings, the charge asymmetry sets a lower limit on the mass of the color-octet, while an upper bound can be set thanks to the invariant mass distribution.

\section{The charge asymmetry at the LHC}

The LHC is a proton-proton machine, thus its initial state is symmetric and the forwardbackward asymmetry vanishes. However, it is still possible to find a charge asymmetry in selected kinematic regions. We define a central asymmetry [1]:

$$
A_{C}\left(y_{C}\right)=\frac{N_{t}\left(|y| \leq y_{C}\right)-N_{\bar{t}}\left(|y| \leq y_{C}\right)}{N_{t}\left(|y| \leq y_{C}\right)+N_{\bar{t}}\left(|y| \leq y_{C}\right)}
$$

It obviously vanishes if the whole rapidity spectrum is integrated, while a non-vanishing asymmetry can be obtained over a finite interval of rapidity.

At the LHC most of the events ( $70 \%$ at $7 \mathrm{TeV})$ are gluon-gluon fusion, which are symmetric. However, it is possible to enhance the asymmetry through a cut on the top-antitop invariant mass, $m_{t \bar{t}}>m_{t \bar{t}}^{\min }$, because that region of the phase space is more sensitive to the quark-antiquark induced events rather than the gluon-gluon ones.

There are some models of resonances which do not produce a charge asymmetry at LO in the inclusive process. This happens if any of the axial-vector couplings vanishes, like, for instance, in some models of Kaluza-Klein gluon, where $g_{A}^{q}=0$ for light flavours. Nonetheless, a charge asymmetry is generated in the exclusive $t \bar{t}+$ jet process: it is now a leading order effect and, besides the contribution of gluon bremsstrahlung from fermions, also the gluon emission from the exchanged particle contributes to the asymmetry.

In Fig. 3 we show the result of our analysis for a scenario of a typical Kaluza-Klein gluon excitation [11]. We have calculated the central asymmetry and the luminosity needed to reach a 
statistical significance of 5 as a function of the cut on the invariant top-antitop quark mass. We find that there is a minimum in this luminosity for relatively low cuts: this is a non-trivial result as it means that relatively low energetic top quarks can already generate a measurable asymmetry. This is an advantage, because very boosted top quarks are difficult to distinguish from jets initiated by light quarks.
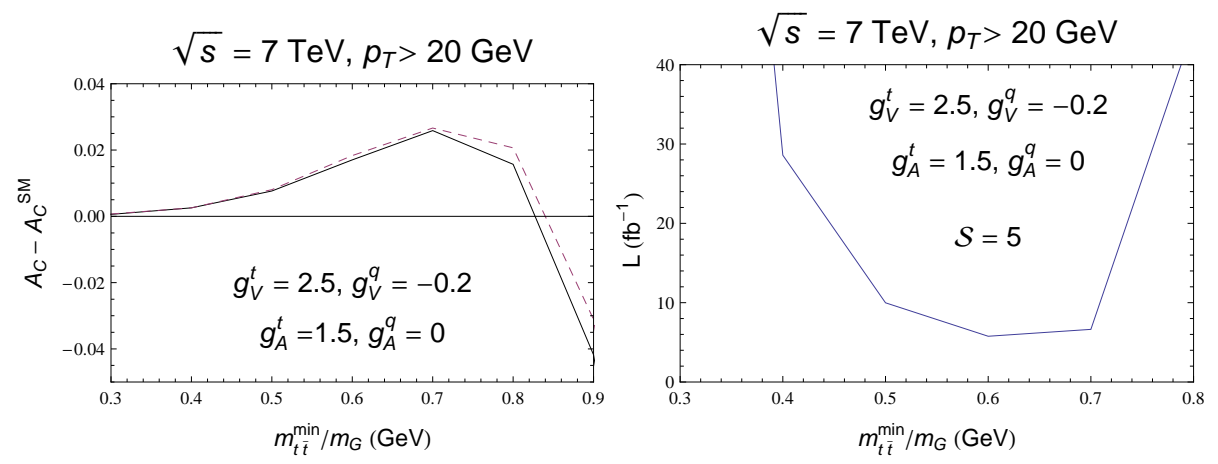

Figure 3: Central charge asymmetry and luminosity to obtain a statistical significance $\mathscr{S}=5$ at the LHC, as a function of $m_{t \bar{t}}^{\min }$ for $\sqrt{s}=7 \mathrm{TeV}$. The dashed line represent the contribution of the terms proportional to $d_{a b c}^{2} . m_{G}=1.5 \mathrm{TeV}$.

\section{Aknowledgements}

This work is supported by Grant No. FPA2007-60323, by Grant No. CSD2007-00042, by Grant No. PROMETEO/2008/069, and by Contract No. MRTN-CT-2006-035482.

\section{References}

[1] O. Antuñano, J. H. Kühn and G. Rodrigo, Top Quarks, Axigluons and Charge Asymmetries at Hadron Colliders, Phys. Rev. D 77014003 (2008) [arXiv:0709.1652 [hep-ph]].

[2] L. M. Sehgal and M. Wanninger, Forward-backward asymmetry in two jet events: signature of axigluons in proton-antiproton collision, Phys. Lett. B 200211 (1988).

[3] J. H. Kühn and G. Rodrigo, Charge asymmetry of heavy quarks at hadron colliders, Phys. Rev. D 59, 054017 (1999) [arXiv: hep-ph/9807420]; Charge asymmetry in hadroproduction of heavy quarks, Phys. Rev. Lett. 81, 49 (1998) [arXiv: hep-ph/9802268].

[4] T. Aaltonen et al. [CDF Collaboration], Forward-Backward Asymmetry in Top Quark Production in ppbar Collisions at $\sqrt{s}=1.96$ TeV, Phys. Rev. Lett. 101202001 (2008) [arXiv: 0806.2472 [hep-ex]].

[5] V. M. Abazov et al. [D0 Collaboration], First measurement of the forward-backward charge asymmetry in top quark pair production, Phys. Rev. Lett. 100142002 (2008) [arXiv: 0712.0851 [hep-ex]].

[6] G. L. Strycker et al. [CDF Collaboration], Measurement of the Forward-Backward Asymmetry in Top Pair Production in 3.2/fb of ppbar collisions at sqrt(s)=1.96 TeV Conf. Note 9724 (March 2009). 
[7] G. Rodrigo, Axigluon signatures at hadron colliders, POS (RAD COR 2007) 010 (2008) [arXiv:0803.2992 [hep-ph]].

[8] P. Ferrario and G. Rodrigo, Massive color-octet bosons and the charge asymmetries of top quarks at hadron colliders Phys. Rev. D 78094018 (2008) [arXiv: 0809.3354 [hep-ph] ].

[9] T. Aaltonen et al. [CDF Collaboration], First Measurement of the t $\bar{t}$ Differential Cross Section $d \sigma / d M_{t \bar{t}}$ in $p \bar{p}$ Collisions at $\sqrt{s}=1.96$ TeV, Phys. Rev. Lett. 102, 222003 (2009) [arXiv:0903.2850 [hep-ex]].

[10] P. Ferrario and G. Rodrigo, Constraining heavy colored resonances from top-antitop quark events, Phys. Rev. D 80051701 (2009) [arXiv:0906.5541 [hep-ph] ].

[11] P. Ferrario and G. Rodrigo, Heavy colored resonances in top-antitop + jet at the LHC, JHEP 1002, 051 (2010) [arXiv:0912.0687 [hep-ph] ]. 